\title{
Correlation detector based on a FPGA for ultrasonic sensors
}

\author{
J. Ureña*, M. Mazo, J.J. García, Á. Hernández, E. Bueno \\ Department of Electronics, University of Alcalá, Escuela Politécnica, Campus Universitario s/n., Alcalá de Henares, Madrid 28871, Spain
}

Received 30 September 1998; received in revised form 27 November 1998; accepted 11 January 1999

\begin{abstract}
In the field of mobile robots ever more frequent use is being made of ultrasonic sensors composed of several transducers carrying out readings simultaneously and in a coordinated way. These sensors can read not only the distances but also listening angles, and they can also determine the reflector type (i.e. edge, plane or corner). To this end it is especially important that, after each emission of a pulse of ultrasound, each transducer can accurately detect the arrival of the echoes (providing accurate time-of-flight (TOF) readings). Another desirable feature is that the transducers be capable of discriminating between the echoes of simultaneous emissions (avoiding problems of crosstalk). This article describes the design and development of a correlation detector (implemented on a low-cost electronic system) allowing for both functions. (C) 1999 Elsevier Science B.V. All rights reserved.
\end{abstract}

Keywords: FGPA-based correlation detector; Pulse compression; Intelligent sensor; Ultrasonic sensor; Mobile robot

\section{Introduction}

Proposed techniques for improving a transducer's capacity to measure accurate times of flight (TOFs) normally involve processing of the echo signal along similar lines to those used in radar and sonar [1]. The great drawback of this approach is that each transducer must necessarily be equipped with a processing system for carrying out all necessary operations. This may significantly increase the complexity of the overall system, making it more difficult to set up and cutting down one of the great advantages of ultrasonic sensory systems: their relatively low cost and simplicity. Further the calculating time of the algorithm used must be as low as possible, to avoid further delays in obtaining readings. All these aspects call for very careful choice of algorithm and hardware to be used.

In previous studies more stress has usually been laid on the algorithms used rather than the way of implementing them so that they may viably be extended to a complete sonar system for a Mobile Robot (MR), which may comprise up to twenty transducers. In [2] it was proposed that a network of transputers be used for calculating the correlation between the signal received and an "idealised echo signal" (belonging to an isolated reflector in the absence of any noise). Working from the signal obtained, a peak search is made at the correlator output, rejecting

\footnotetext{
* Corresponding author. Tel.: +34-1-8856544; fax: +34-1-88546591.

E-mail address: urena@depeca.alcala.es (J. Ureña)
}

those lesser to a certain threshold and those that are insufficiently separated.

A similar algorithm can be seen in [3] based on a correlation of the signal emitted (suitably modulated) with the signal received and a subsequent peak search. To implement these into a single transducer, a PC with an A/D card was used, to be replaced in the future by a DSP.

In the sensor proposed in [4] the correlation of the signal received is effected, not with one, but with 20 "standard", signals corresponding to 20 echoes representing different reflectors and different reception angles. The processing time in this case for each reflector is one second, using a PC-386 together with a data capture system.

The process was also carried out with a PC in the sensor analysed in [5]. In this case, however, pseudo-random codes were used to encode the signals emitted simultaneously by several transducers, so that the echoes could be differentiated by the receivers.

The work presented in this article uses an algorithm based on digital modulation of the signal emitted and the correlation thereof with the echo signal received. The implementation of the algorithm is effected by a low-cost FPGA, type Xilinx 4005E [6] so that the system may be easily set up for all the transducers used in a single sonar module [7]. The final system must allow for the following:

1. Ease of combining one transducer with another, so that they may make up more complex sensory modules.

2. High sensitivity, enabling the detection of echoes from small objects or those set apart from the transducer axis. 




Fig. 1. Echo detected after rectification, integration and thresholding.

3. Ability to detect multiple echoes in a single reading.

4. High accuracy in the determination of the TOFs (distances determined with sub-millimetre accuracy).

5. Possibility of emitting simultaneously with two transducers, the receivers then being able to discriminate which transducer the echoes are emitted by.

Later, in Section 2a description is given of the modifications carried out on the basic POLAROID system [8] and the hardware solution adopted for each one of the parts the algorithm has been divided into. In Section 3 there is a description of how the system has been set up for utilisation with two simultaneous emitters. Finally, in Section 4, some results are given for the system as actually implemented.

\section{Improvement of the transducer electronic system}

A basic transducer, such as that of POLAROID, employing integration and thresholding techniques of the echo signal, gives an accuracy (see Fig. 1) that may suffice for many MR applications requiring only distance measurement but not for the determination of reception angles nor for a correct classification of the detected reflectors.

\subsection{Proposed electronic system}

To improve the transducer's capacity of measuring TOFs accurately, the echo signal is processed along similar lines to those used in radar. The great drawback is that each transducer must necessarily be equipped with a processing system for carrying out all necessary operations. This may significantly increase the complexity of the total system, making its assembly more difficult and cutting down some of the great advantages of ultrasonic sensory systems: their relatively low cost and simplicity. To avoid this, the algorithms have been implemented on a specific digital system, based on FPGA 4005E of Xilinx, the block diagram of which is shown in Fig. 2.

From an external point of view the working of the transducer may be controlled by a micro-controller system, through an interface circuit, using only four digital lines, namely: (a) mode signal (E-R/R); this digital line indicates whether the transducer has to function in EMITTERRECEIVER mode (E-R) or as RECEIVER only (R), (b) Initiating signal (INIT); this signal indicates the moment when the measuring process starts, (c) CLOCK signal: the functioning of the whole system, in its digital processing facet, calls for a single clock synchronising signal and (d) Echo reception signal (VALIDATED ECHO): as the echo signal is received it is processed to determine the arrival times of the various echoes detected. Each time one of the echoes is validated the ECHO VALIDATED signal is turned on for $32 \mu \mathrm{s}$. The system resolution has been established with a minimum TOF difference of $64 \mu \mathrm{s}$ (a distance resolution of about $1 \mathrm{~cm}$ ).



Fig. 2. Electronic system for the transducer. 




Fig. 3. Block diagram for the processing of the received signal.

\subsection{Implemented algorithm}

A practical approach to optimum filtering [3] involves taking the emitted signal as reference and making a crosscorrelation between this and the signal received (see Fig. 3). A peak search is made at the correlator output, the peaks coincide with the arrival of the echoes. From a digital point of view, $y(n)$ is:

$y[n]=e[n] * r[n]=\sum_{k=0}^{N} e[k] \cdot r[n+k]$

where $N$ is the number of samples of the emitted signal, $e[n]$, and $r[n]$ is the received signal.

The ultrasonic transducer used requires binary excitation, so the signal emitted has to be of this type. A binary sequence whose autocorrelation function has a straight lobe is the 13-bit Barker code. The main lobe has a width of one bit and amplitude 13 times higher than the side lobes.

The signal emitted by the transducer must be $50 \mathrm{kHz}$ and last long enough for the energy emitted to allow the detection of echoes from reflectors at a considerable distance. For each bit of the Barker code a symbol has been emitted composed of two periods of the carrier signal (at $50 \mathrm{kHz}$ ). In total $13 \times 40 \mu \mathrm{s}=520 \mu \mathrm{s}$. A two-phase modulation was used, emitting with phase zero if the code bit is 1 , and complemented phase if it is -1 . A separation time equal to the sampling time $(2 \mu \mathrm{s})$ is set between consecutive samples, and the signal emitted can be obtained as the correlation between the symbol used (two periods) and the Barker code sequence (but in this case with consecutive samples separated by a symbol-20 periods), as may be seen in Fig. 4. Note that $e[n]$ is the correlation between $c[n]$ and $b[n]$ :

$e[n]=c[n] * b[n]$.

Fig. 5 shows the implementation on the FPGA of the module for emitting such sequence.

\subsection{Implementation of the correlation and detection of peaks}

The sampling period used in the digitalisation of the signal received is $2 \mu \mathrm{s}$ (sampling at $500 \mathrm{kHz}$ ). In other words, for each sequence emitted there will be $520 / 2=$ 260 samples. The reception of the echo signal is maintained for $32 \mathrm{~ms}$ (to detect distant targets up to about $6 \mathrm{~m}$ ). But the correlation is effected while the signal is being digitised, maintaining only a reception memory capacity of 260 samples (equal to the duration of the signal emitted). The total correlation between the signal emitted and received may be broken down into two successive correlations:

$$
\begin{aligned}
y[n] & =r[n] * e[n]=r[n] *(c[n] * b[n]) \\
& =(r[n] * c[n]) * b[n]=C_{1}[n] * b[n] .
\end{aligned}
$$

\subsubsection{First correlation $(C 1[n])$}

Between the signal received, once digitised, and the sequence corresponding to a symbol (20 samples). As the latter only takes on the values +1 and -1 , the correlation is simplified as only additions and subtractions are necessary (not products). The process will involve taking twenty samples of the signal received and, in units of five, adding or subtracting them according to the following sequence: $\{+++++-----+++++----\}$. Fig. 6 shows a block diagram of the circuit for this process. The algorithm
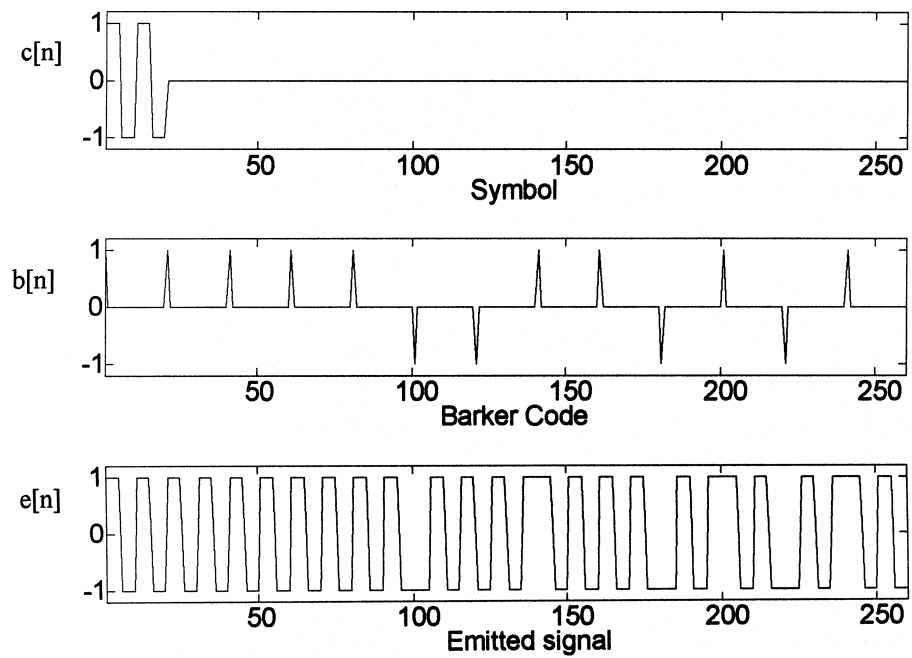

Fig. 4. Generation of the emitted signal. 


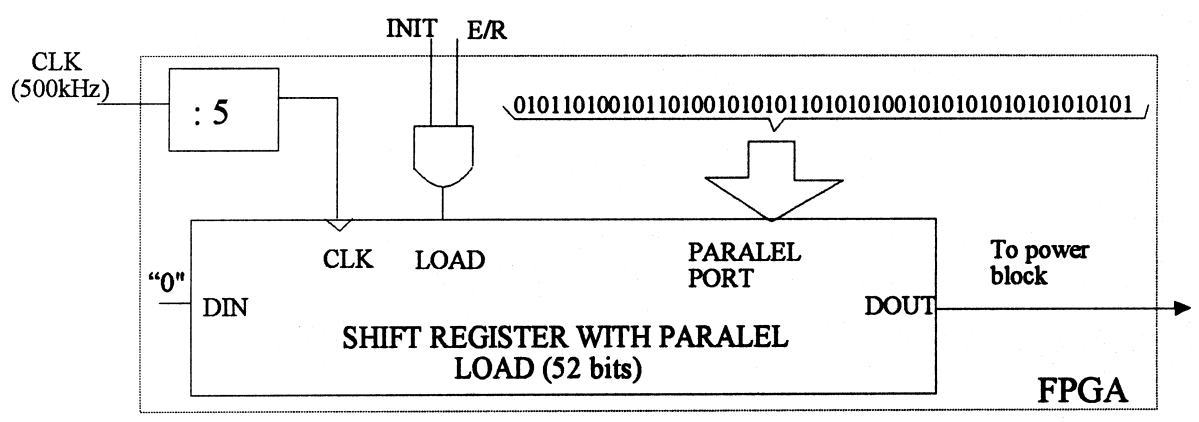

Fig. 5. Block diagram to generating the emitted sequence.

implemented, in a iterative way, is:

$$
\begin{aligned}
C_{1}[n]-C_{1}[n-1]= & -r[n-1]+2 \cdot r[n+4]-2 \cdot r[n+9] \\
& +2 \cdot r[n+14]-r[n+19] .
\end{aligned}
$$

\subsubsection{Second correlation}

The result of the first correlation must be correlated with the Barker code, $b[n]$. As this code is normalised at values +1 or -1 , this second correlation $\left(C_{2}[n]=y[n]\right)$ can be performed by doing:

$$
\begin{aligned}
C_{2}[n]= & \sum_{k} b[n] \cdot C_{1}[n+k]=\sum_{k=0}^{4} C_{1}[n+20 \cdot k] \\
& -\sum_{k=5}^{6} C_{1}[n+20 \cdot k]+\sum_{k=7}^{8} C_{1}[n+20 \cdot k] \\
& -C_{1}[n+9 \cdot 20]+C_{1}[n+10 \cdot 20]-C_{1}[n+11 \cdot 20] \\
& +C_{1}[n+12 \cdot 20] .
\end{aligned}
$$

In each sampling period the result of the first correlation is taken to an external RAM memory whose addresses are accessed by a pointer-counter in cyclical mode. The capacity of this memory is $13 \times 20=260$ words. The block diagram for this second correlation is shown in Fig. 7. During the sampling period $(2 \mu \mathrm{s})$ a 16-state sequential process is synchronised by a frequency clock 16 times higher $(8 \mathrm{MHz})$. In these states (see Fig. 8 ) the following operations are carried out:

State 0 While the value of the previous correlation is stored in the relevant memory address (picked out by the counter acting as pointer), it is stored in an accumulator (this is the sample associated with the 13th bit of the Barker code).

States 1-12 At the beginning of every state, twenty is added to the pointer value existing in the previous one. The contents of the new direction pointed are introduced in the accumulator, with a sign plus or minus according to the 1-12 bits of the Barker code sequence.

States 13-15 These are used for peak detector operations with the last correlation result.

The combinational logic block performs the function shown in Table 1.

\subsubsection{Peak detector}

A comparator is used so that only output values of the second correlation higher than a certain threshold (externally selected) are taken into account. When this obtains, this instant is stored and is definitely validated as



Fig. 6. Block diagram for the first correlation. 


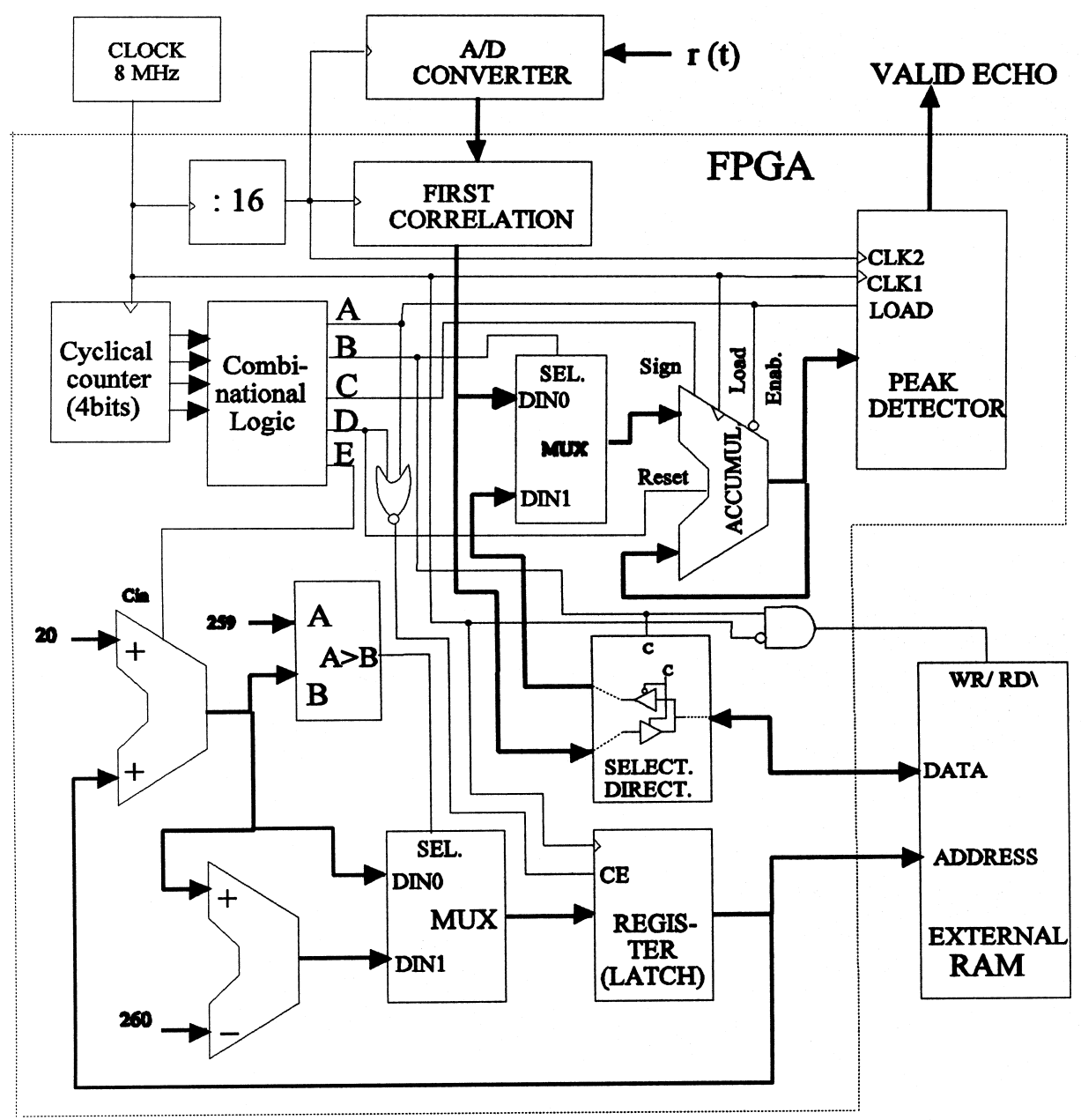

Fig. 7. Circuit for the second correlation.

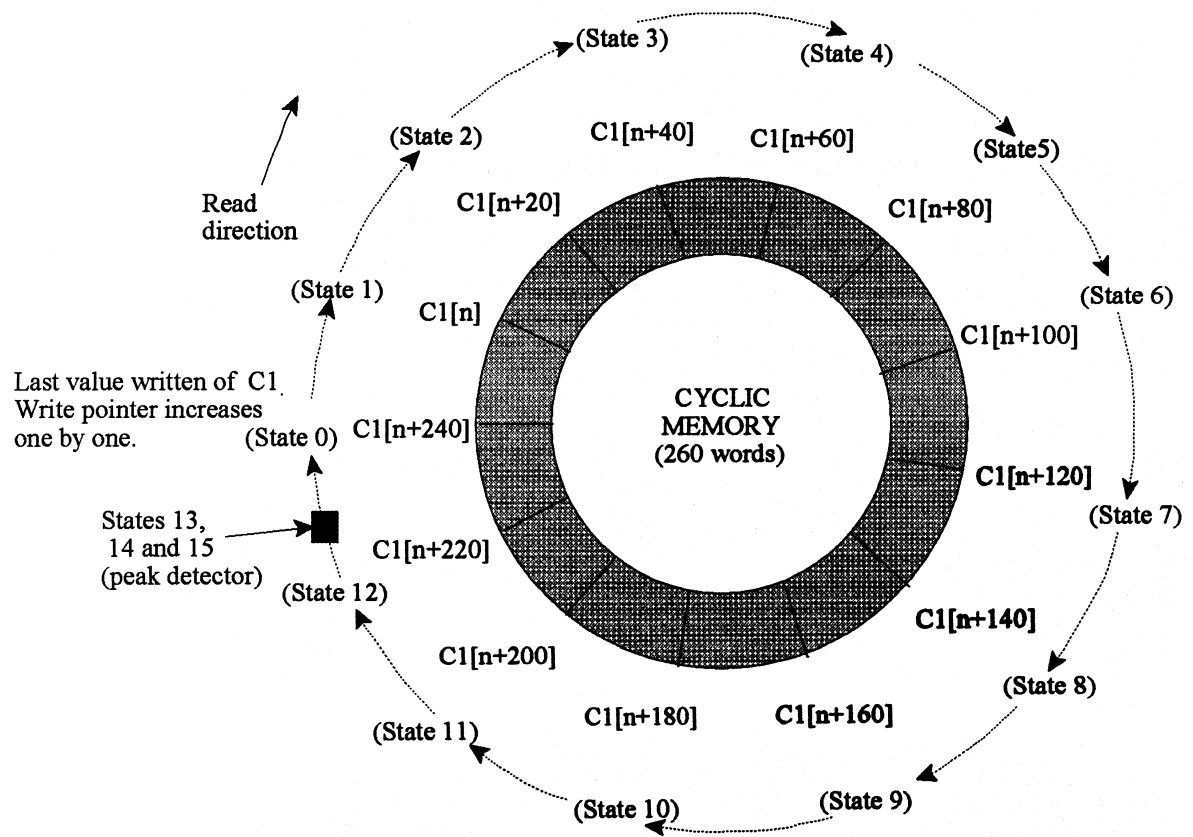

Fig. 8. Cyclical access to the external RAM with a 16-states counter. 
Table 1

Combinational function (in Fig. 6)

\begin{tabular}{llllll}
\hline States & A & B & C & D & E \\
\hline 0 & 0 & 1 & 1 & 0 & 0 \\
$1,2,3,4,5,8,9,11$ & 0 & 0 & 1 & 0 & 0 \\
$6,7,10$ & 0 & 0 & 0 & 0 & 0 \\
12 & 0 & 0 & 0 & 0 & 1 \\
13 & 1 & 0 & $x$ & 0 & $\mathrm{x}$ \\
14,15 & 0 & 0 & $\mathrm{x}$ & 1 & $\mathrm{x}$ \\
\hline
\end{tabular}

an echo reception moment if there is no higher value in the 16 following samples (and at the end of same). In other words two echoes separated (in flight time) by less than $32 \times 2 \mu \mathrm{s}=64 \mu \mathrm{s}$ (tantamount to about $10 \mathrm{~mm}$ of reflector separation) will never be distinguished. In practice, this "minimum separation between echoes" can be externally configured to be between 64 and $2048 \mu \mathrm{s}$.

When a valid peak is detected the VALID ECHO output is activated (at a high level) during $32 \mu \mathrm{s}$ (guaranteeing that during at least $32 \mu \mathrm{s}$ it will not again be activated). It should be pointed out that from the moment an echo is received until it is actually validated with the corresponding signal there is a systematic processing time of 260 periods for both correlations and 32 periods for the echo validation, i.e., $292 \times 2 \mu \mathrm{s}=584 \mu \mathrm{s}$. The external system must subtract this time from the value actually measured. Fig. 9 shows the block diagram of the peak detector.
Table 2

System differences according to the frequency used

\begin{tabular}{lcc}
\hline Parameter & System 1 & System 2 \\
\hline Sampling period & $2 \mu \mathrm{s}$ & $1,78 \mu \mathrm{s}$ \\
Sampling frequency & $500 \mathrm{kHz}$ & $562.5 \mathrm{kHz}$ \\
Ultrasound emission frequency & $50 \mathrm{kHz}$ & $56.25 \mathrm{kHz}$ \\
Duration of pulse emitted & $520 \mu \mathrm{s}$ & $462.8 \mu \mathrm{s}$ \\
Minimum echo separation & $64 \mu \mathrm{s}$ & $57 \mu \mathrm{s}$ \\
Echo validation pulse & $32 \mu \mathrm{s}$ & $28.5 \mu \mathrm{s}$ \\
\hline
\end{tabular}

\section{Extension to two emitters}

The possibility of emitting simultaneously with two transducers involves linking up each transducer to another system identical to the one described, but governed by a higher original clock signal (about $9 \mathrm{MHz}$ for example), so that sampling frequency is $562.5 \mathrm{kHz}$ (sampling period of $1.77 \mu \mathrm{s}$ ). The signal generated for emission will thus be composed by frequency symbols equal to $56.25 \mathrm{kHz}$, to which the transducer adequately responds both in emission and reception. Table 2 shows the most important variable changes brought about by this frequency change.

If the two transducers emit simultaneously, one at $50 \mathrm{kHz}$ and the other at $56.25 \mathrm{kHz}$, and then the echo signal is digitised by all the receivers with both systems, each one provides the echoes corresponding to one emission or the other.

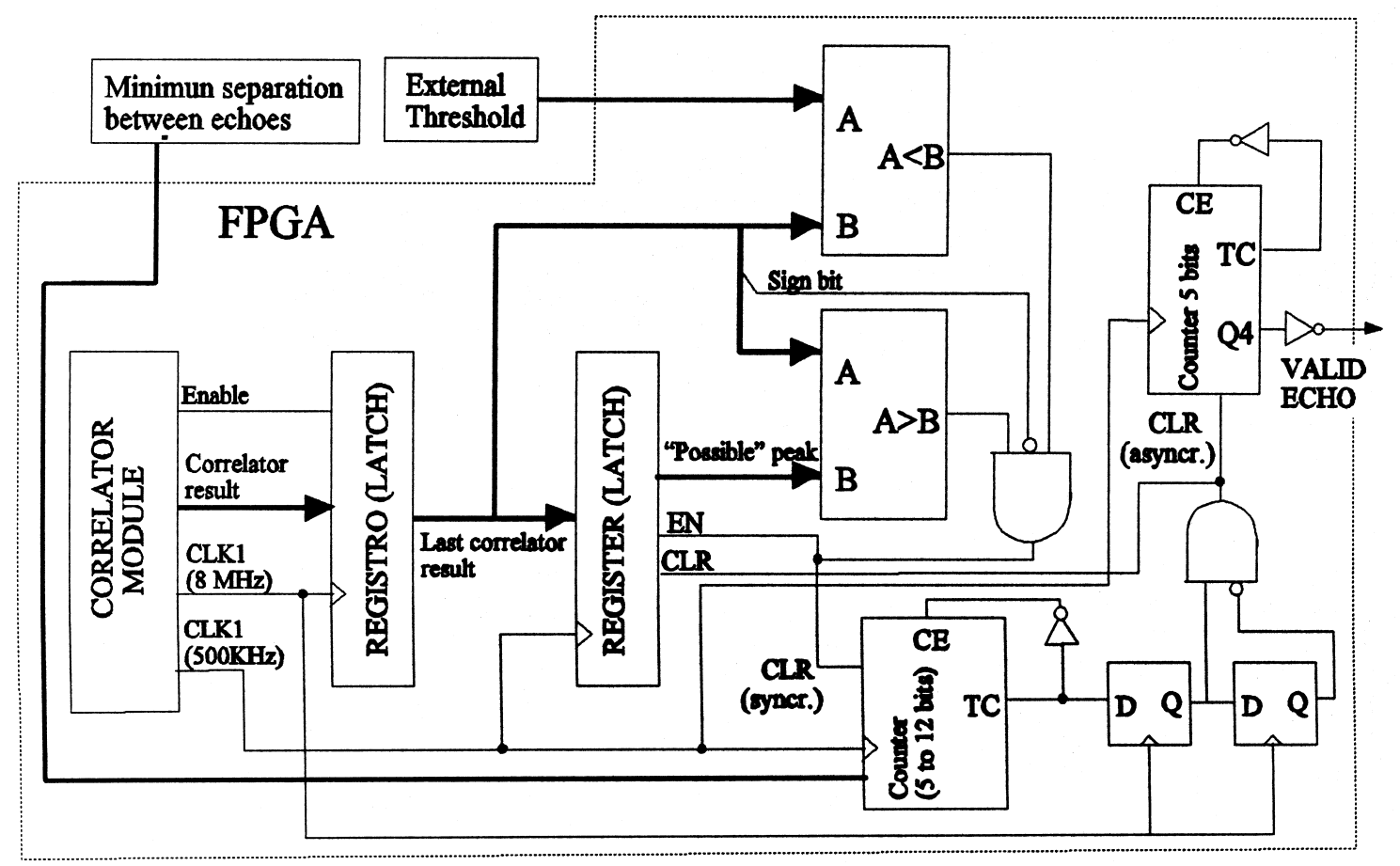

Fig. 9. Block diagram for the peak detector. 


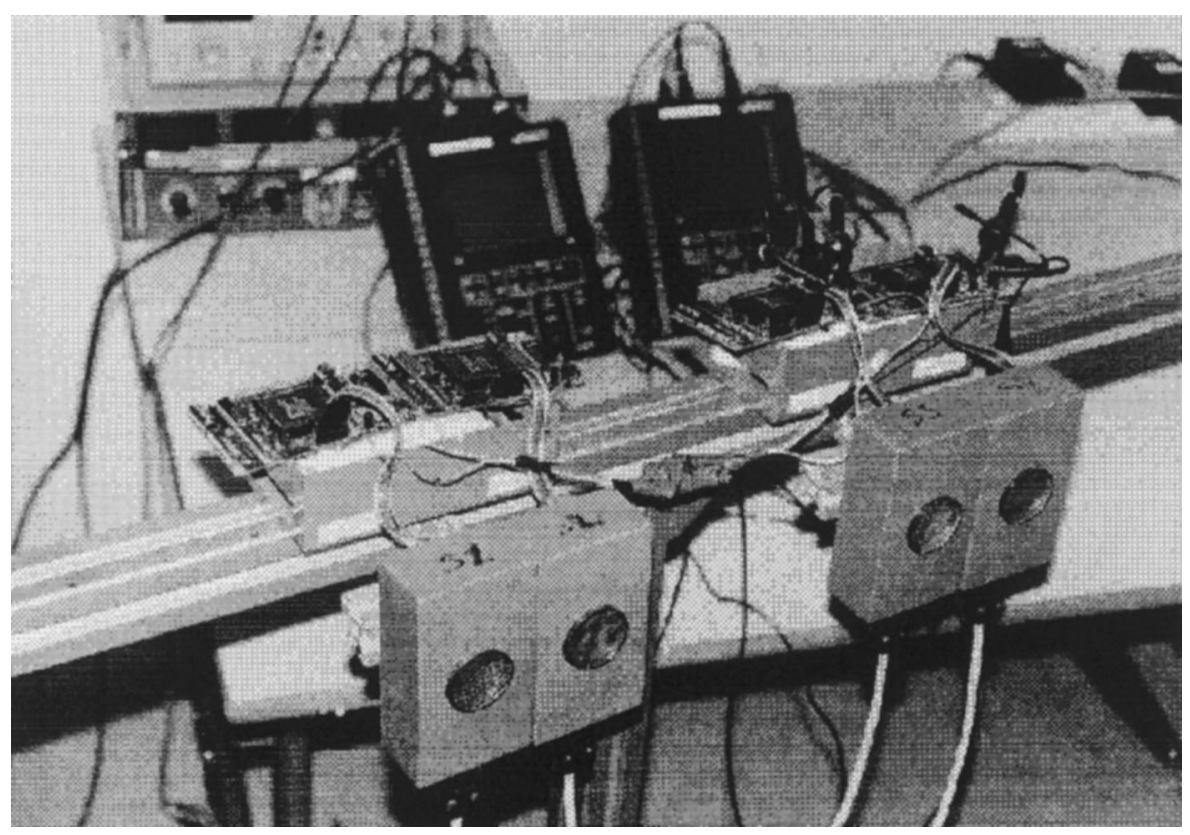

Fig. 10. View of four transducers with the electronic boards.

\section{Results}

A four-transducer sensory system [9] has been implemented (see Fig. 10) to carry out some experimental results. These results have proved the enhancement of the system obtaining a time-of-flight precision of 2-4 $\mu$ s for distances less than $3 \mathrm{~m}$.

Fig. 11 shows the echo signals (analogue and once processed) for the case of a single isolated reflector at $60 \mathrm{~cm}$ from the transducer. It should be noted that the analogue signal received initially takes in the emission excitation itself. After a time of $3520 \mu$ s has passed, the echo pulse reception is initiated. The digital echo-validation signal is delayed for a minimum time of $586 \mu \mathrm{s}$ (the time for the capture, processing and validation of the echo). Meanwhile, throughout this last interval of time the echo pulse has been received and at the same time processed.

The transducer's multi-echo detection capacity is reflected by the capture effected in a more complex environment, as shown in Fig. 12. In this case five echo pulses corresponding to five different reflectors are detected and validated even when these pulses are partly superimposed. It should be noted that in the case of such environments it might be usual to detect pulses coming from multiple rebounds. This confirms the importance of limiting the detection to a certain number of pulses (the first ones received), as the likelihood thereafter increases of receiving echoes from multiple reflections.

The adjustment of the external threshold was empirically effected without great difficulties: excessively low values provoke the validation of echoes at undesirable moments
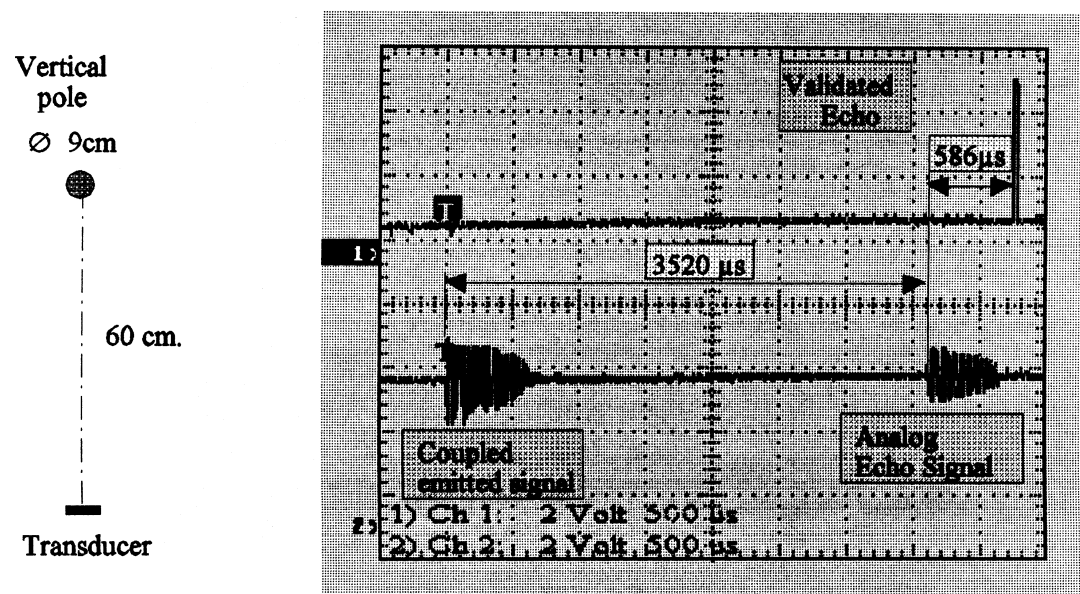

Fig. 11. Echo detection for an isolated reflector. 

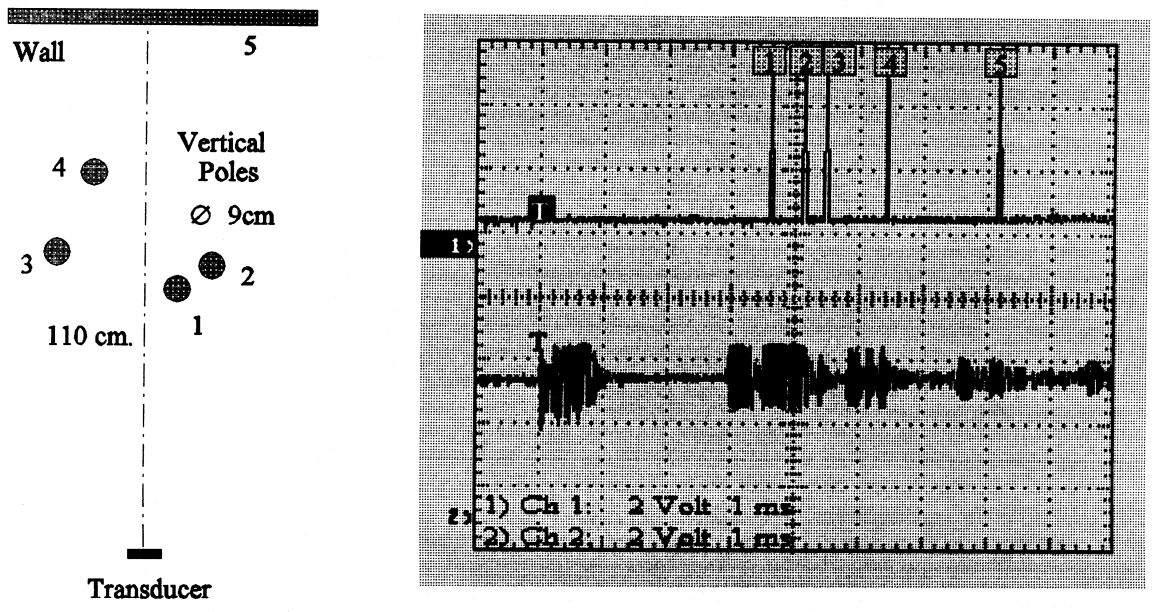

Fig. 12. Multi-echoes detection (five reflectors).

(presence of noise or side lobes at the correlator output), while high values block the echo detection when the analogue signal is relatively poor. Minimum echo separation has been increased to $1024 \mu \mathrm{s}$ (instead of the minimum value of $64 \mu \mathrm{s})$ in cases where the detection of very close obstacles is not necessary; this avoids the validation of consecutive multiple echoes coming from the same reflector.

\section{Acknowledgements}

This work has been carried out thanks to the grants received from the CICYT (Interministerial Science and Technology Committee -Spain-) projects TAP94-0656003-01 and IER96-1957-C03-01.

\section{References}

[1] S.A. Hovanessian, Radar System Design and Analysis, Artech House, Inc, Norwood, 1984.

[2] K. Audeanert, H. Peremans, Y. Kawahar, J. van Campenhout, Accurate ranging of multiple objects using ultrasonic sensors, Proceedings of IEEE International Conference on Robotics and Automation, Nice, 1992, pp. 1773-1738.

[3] H. Hamadene, E. Colle, Optimal estimation of the range for MRs using ultrasonic sensors, SICICA '97, 1997, pp. 141-146.

[4] L. Kleeman, R. Kuc, Mobile robot sonar for target localisation and classification, The International Journal of Robotics Research 14 (4) (1995) 295-318.

[5] J. Klaus-Werner, B. Markus, M. Markus, Using pseudo-random codes for mobile robot sonar sensing, Third IFAC Symposium on Intelligent Autonomous Vehicles (IAV '98), Madrid, 1998, pp. 231-236.

[6] Xilinx Corporation, FPGAs, Technical Handbook, 1995.

[7] M. Mazo, F.J. Rodríguez, M.A. Sotelo, J. Ureña, J.C. García, J.L. Lázaro, F. Espinosa, Automation of an industrial fork lift truck, guided by artificial vision, ultrasonic and infrared sensors in open environments, International Conference on Field and Service Robots FSR '97, Canberra, 1997, pp. 192-199.
[8] Polaroid Corporation, Ultrasonic Ranging Systems, Manual and Handbook, 1991.

[9] J. Ureña, M. Mazo, J.J. García, E. Bueno, Ultrasonic sensor module for an autonomous industrial vehicle, Third IFAC Symposium on Intelligent Autonomous Vehicles (IAV '98), Madrid, 1998, pp. 219-224.



Jesús Ureña received the Electronic Engineering and Telecommunication Engineering degrees from Polytechnical University of Madrid (Spain) in 1986 and 1992, respectively; and the $\mathrm{Ph} . \mathrm{D}$. degree in Telecommunications from the University of Alcalá (Spain) in 1998. Since 1986 he is a lecturer at the Electronic Department of the University of Alcalá. During this period he has collaborated in several educational and research projects in the areas of electronic control and electronic and sensorial systems for mobile robots.



Manuel Mazo received the Electronic Engineering degree in 1976, the Telecommunications Engineering degree in 1982, and Ph.D. degree in Telecommunications in 1982, all from the Polytechnic University of Madrid (Spain). Currently, he is Professor in the Department of Electronics at the University of Alcalá. His areas of research are multi-sensor (ultrasonic, infrared and artificial vision) integration applied to mobile robots, man/ machine cooperation in the field of technical aid for people with disabilities (wheelchairs for physically disabled people), and electronics control systems. 

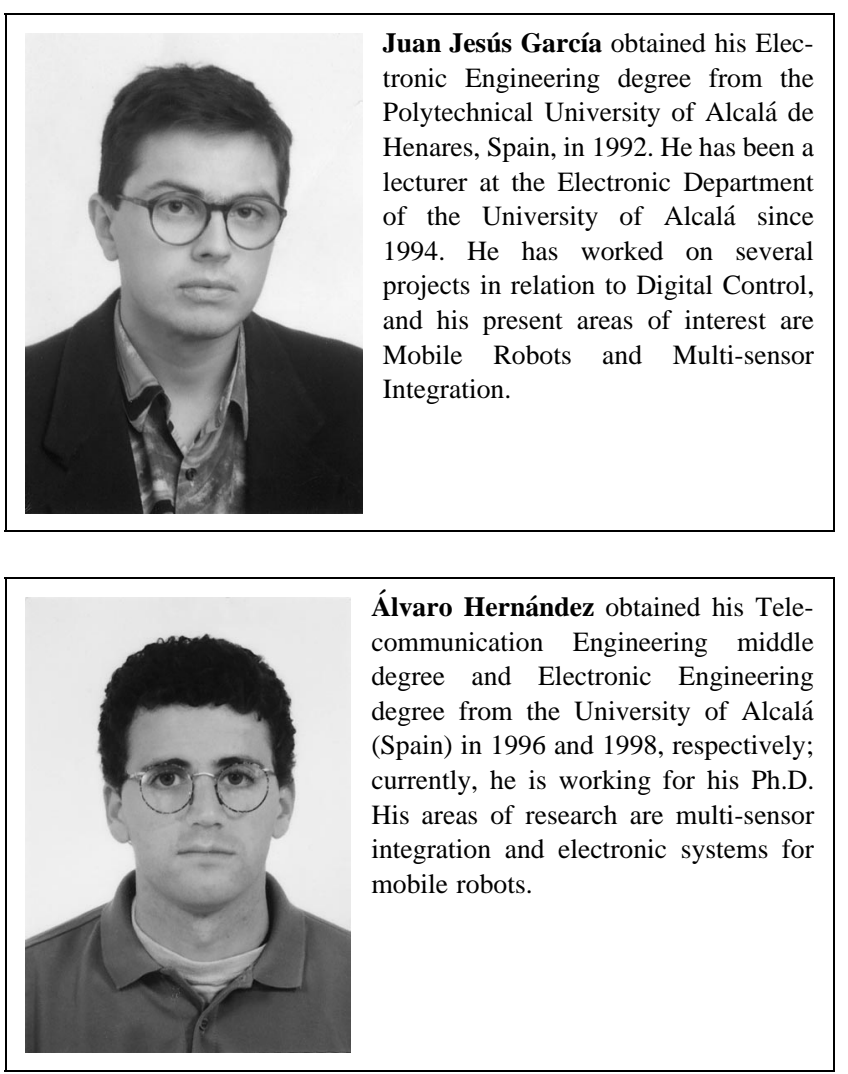



